\title{
Epiluminescence Image Processing for Melanocytic Skin Lesion Diagnosis Based on 7-Point Check-List: A Preliminary Discussion on Three Parameters
}

\author{
Gabriella Fabbrocini ${ }^{*}, 1$, Giovanni Betta ${ }^{2}$, Giuseppe Di Leo ${ }^{3}$, Consolatina Liguori $^{3}$, Alfredo Paolillo ${ }^{3}$, \\ Antonio Pietrosanto ${ }^{3}$, Paolo Sommella ${ }^{3}$, Orsola Rescigno ${ }^{1}$, Sara Cacciapuoti ${ }^{1}$, Francesco Pastore ${ }^{1}$, \\ Valerio De Vita ${ }^{1}$, Ines Mordente ${ }^{1}$ and Fabio Ayala ${ }^{1}$ \\ ${ }^{I}$ Dept of Systematic Pathology - Section of Dermatology. University Federico II of Naples, Italy \\ ${ }^{2}$ Dept of Automation, Electromagnetism, Computer Science and Industrial Mathematics (DAEIMI), University of \\ Cassino, Italy \\ ${ }^{3}$ Dept of Information and Electrical Engineering (DIIIE), University of Salerno, Italy
}

\begin{abstract}
Epiluminescence microscopy (ELM) is a non-invasive technique used to enhance visualization of microscopic structures of pigmented lesions for the early detection of melanoma. The 7-point check-list is a diagnostic method that requires the identification of only seven dermoscopic criteria, defining the image through the use of algorithms. This paper describes an experimental automated diagnosis set-up of melanocytic skin lesions through an image processing methodology focused on finding the presence of different epiluminescence parameters. In this paper the image processing set-up allows the automatic detection of some specific dermoscopic criteria. We analyze the blue whitish veil, the regression, and the irregular streaks. The procedure developed was tested by considering a set of about 200 ELM images. A good concordance between ELM 7-point checklist parameters detected and the new method of image processing was achieved by kappa analysis. Although ELM doesn't substitute histological evaluation, it could be a reliable instrument to enhance clinical accuracy of skin pigmented lesions diagnosis.
\end{abstract}

Keywords: Epiluminescence, 7-point check-list, blue whitish veil, regression structures, irregular streaks, image processing, melamoma diagnosis.

\section{INTRODUCTION}

Nowaday the possibility to increase the accuracy of diagnosis of melanoma is one of the most important tool to reduce the mortality rate of this tumor. The aim of prevention campaigns is to increase public awareness of early warning signs The possibility of new diagnostic methodologies and algorithms can contribute to perform an earlier diagnosis and to reduce the metastatic risk. Investigations have shown that the curability rate of thin melanomas $(<1 \mathrm{~mm})$ is between $91.8 \%$ and $98.1 \%$ [1]. Epiluminescence microscopy (ELM; in vivo cutaneous surface microscopy, dermatoscopy, dermoscopy and magnified oil immersion diascopy) is an in vivo, non invasive technique that has disclosed a new dimension of the clinical morphologic features of pigmented skin lesions, using different incident light magnification systems with an oil immersion technique [2, 3]. Results of previous studies demonstrated that ELM improves accuracy in diagnosing pigmented skin lesions from $10 \%$ to $27 \%$ than clinical diagnosis by the naked eye [4]. Three diagnostic models with similar reliability have become

*Address correspondence to this author at the Dept of Systematic Pathology, Section of Dermatology. University Federico II of Naples, Via Pansini, 580131 Naples, Italy; Tel: 0039-0817464386; Fax: 00390817462442; E-mail: gafabbro@unina.it

The work was done in Naples and Salerno (Campania Region) and Cassino (Lazio Region), Italy. more widely accepted by clinicians: (i) pattern analysis, which is based on the "expert" qualitative assessment of numerous individual ELM criteria, (ii) the ABCD-rule of dermato-scopy which is based on a semi-quantitative analysis of the following criteria: asymmetry (A), border (B), color (C) and different dermatoscopic (D) structures and (iii) the ELM 7-point checklist scoring diagnosis analysis, proposed by Argenziano et al. [5] defining only seven standard ELM criteria: Atypical pigment network, Bluewhitish veil, Atypical vascular pattern, Irregular streaks, Irregular pigmentation, Irregular dots/globules, Regression structures. The ELM 7-point checklist provides a simplification of standard pattern analysis and, if compared to $\mathrm{ABCD}$, allows less experienced observers to achieve higher diagnostic accuracy values [6].

Despite the simplification of the ELM criteria, all the algorithms described have showed higher reliability if they were based on a quantitative system [7]. Consequently, the early diagnosis of skin cancer using digital imageprocessing methods, based on a semiquantitative scale, is a very important issue. Some automated systems, based on ABCD algorithm, have been developed. Ganster et al. [8] developed an automated melanoma recognition system but only 21 parameters were extracted from images. Schmid and Schmid-Saugeon $[9,10]$ proposed a color based segmentation scheme without extracting features. Umbaugh and colleagues [11] developed an automatic color segmentation algorithm with application to skin tumor feature identificat- 
ion. Grana et al. define a new algorithm based on the Catmull-Rom spline method and the computation of the gray-level gradient of points extracted by interpolation of normal direction on spline points was employed [12].

A growing interest has developed in the last decade in the automated analysis of digitized images obtained by epiluminescence microscopy techniques to assist clinicians in differentiating early melanoma from benign skin lesions [13].

Nevertheless, so far, no automated systems have yet been based on the 7-point checklist. From these considerations, and from previous experiences in digital processing of medical images [14-17], the authors have tackled the problem of defining suitable image processing algorithm implementing the 7-point checklist (3). It would be suitable to develop an automatic system that can validate the identification of 7 parameters on each lesion observed by clinicians. Computerized identification could reduce the false positive or false negative clinical diagnosis because it adds a quantitative observation to the "clinical eye observation". In a study of 2003, Hoffmann et al, reported that the combination of clinician and computer may potentially increase the accuracy of PSL (pigmented skin lesions) diagnosis. This may result in improved detection of melanoma and a reduction in unnecessary excisions [18].

The digital process equipped system analyzes epiluminescence images in which the following parameters are recorded: "blue-whitish veil", "regression structures", and "irregular streaks".

\section{MATERIALS AND METHODS}

210 digital dermoscopy images, obtained from the CDROM Interactive Atlas of Dermoscopy [19], were observed by epiluminescence microscopy by two different dermatologists (M.S., G.F.) to evaluate the grade of accuracy in the management of 7 point check list algorithm. The database refers both to cutaneous melanomas and (benignant) melanocytic nevi (also including Clark, Spitz, Reed nevi). For each image, the corresponding clinical and histological analyses are available. The images have been extracted in order to obtain a quite homogeneous diagnosis distribution of the cases with respect to the criterion of interest. In particular the Blue Whitish Veil is present in 135 lesions, whereas the Regression structures are evident in 90 ELM images (only in 20 cases both the features are present), finally the irregular streaks are present in 43 images.

After the medical diagnostic the automatic procedure is run. The image segmentation is firstly performed to highlight the pigmeted lesion; then the procedure for the detection of "blue-whitish veil", "regression structures and "irregular streaks" is performed.

\section{Image Segmentation}

The segmentation is the first as well as the most critical skin lesion image processing step. The aim of the segmentation is to extract the lesion border from the skin image. Boundary detection is a difficult problem in dermatoscopic images because the transition between the lesion and the surrounding skin is smooth and difficult to detect accurately, even for a trained dermatologist.
The realized segmentation algorithm for the skin lesion border extraction consists of three steps: (i) colour to monochrome image conversion; (ii) image binarization using an adaptive threshold; (iii) border identification.

(i) In the first step 3 different monochrome images (8 bits per pixel) are obtained from the source image (RGB standard color, 24 bits per pixel) corresponding to the red, green and blue color components, respectively (an example is reported in Fig. 1). Then, the pixel intensity histogram is evaluated, as you can see (Fig. 1) for each component, two modes are typically evident corresponding respectively to the pigmented lesion and the surrounding skin.

(ii) The binarization (i.e. a conversion to a 1-bit/pixel format) of each monochrome image is carried out by thresholding its intensity: the image pixels with gray level lower than the threshold become black whilst the pixels greater than the threshold are set to white. An adaptive threshold is obtained for each monochrome image by adopting the Otsu algorithm [20], which is able to split each bimodal intensity histogram into 2 classes corresponding respectively to the image background (healthy skin) and foreground (pigmented skin). The monochrome image characterized by the wider pigmented skin is adopted to compute the binary mask as reported in the example of Fig. (2b). The lesion appears in white against a black background, except for possible isolated black points in the white skin region. Hence, a morphological closing operator fills isolated black points in the white regions.

(iii) Finally, in order to extract the contour of the lesion, a simple blob-finding algorithm [21] is adopted for the binary image previously obtained: the tracking algorithm collects and sorts out the edges of the black-white image into an ordered list. At this point, the border is superimposed on the color ELM image (Fig. 2c) and displayed for visible inspection to the diagnostician. The binary image is also used in calculating the lesion dimension (number of white pixel) that will be used in the lesion analysis.

\section{Algorithm for the Detection of: Blue-Whitish Veil and Regression}

For the automatic detection of the blue-whitish veil and regression area in digital ELM images a procedure with 2 main stages is applied on the image of the pigmented lesion; (i) the partition of the lesion into its different color components (lesion segmentation) and finally (ii) the recognition of the criterion of interest in the lesion map (region classification).

(i) Starting from the source image and the binary mask, the lesion segmentation stage is carried out with the aim of splitting the internal area into multiple chromatically homogenous regions (the lesion map). The basic idea is the adoption of a suitable multithresholding of the colour image. In particular the following steps are proposed [21]: Principal Component Analysis (PCA) [17]; 2D histogram construction; peaks picking algorithm; histogram partitioning; lesion partitioning. Once the partitioned 2-D is 
(a)
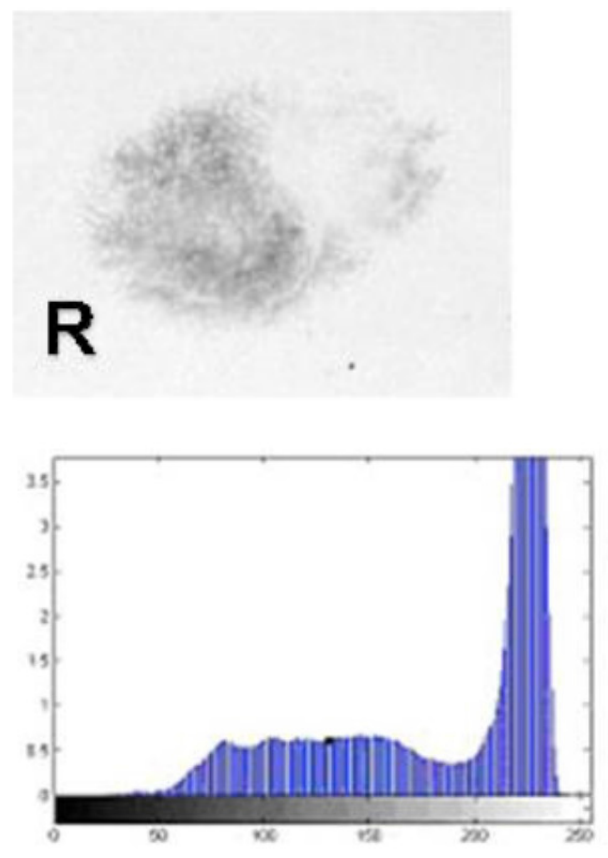

(b)
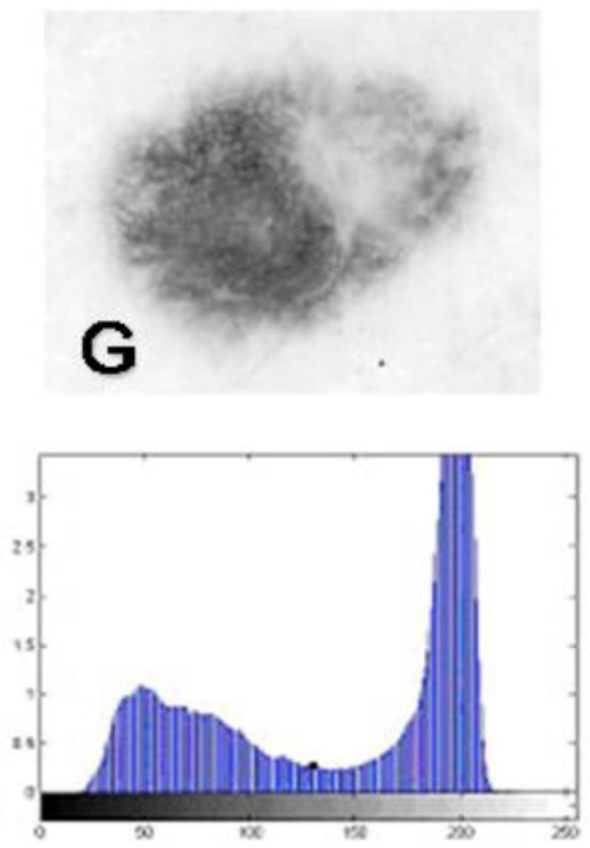

(c)
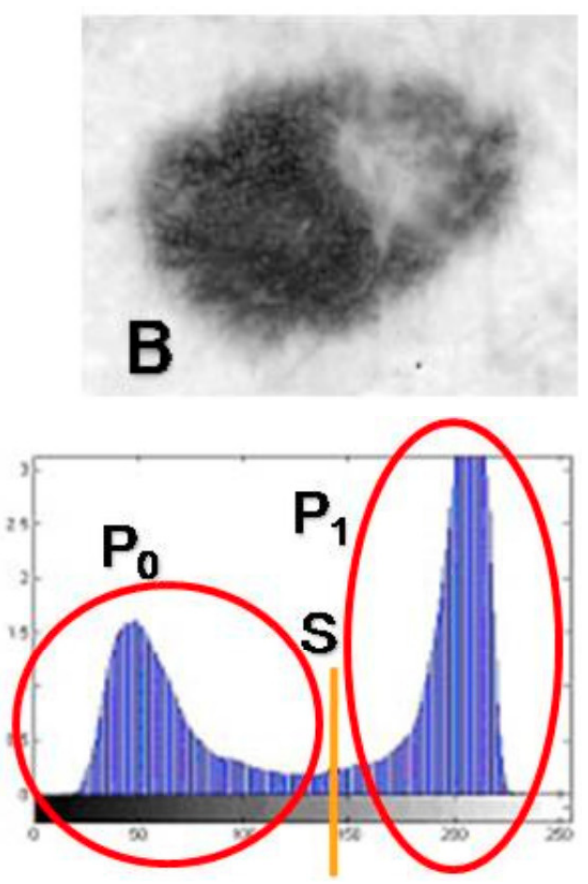

Fig. (1). Monochrome images for Red, Green and Blue Planes and corresponding Intensity Histograms

(a)

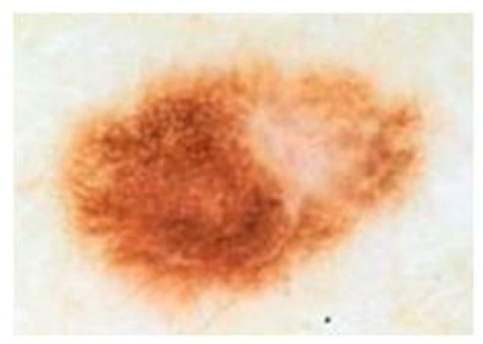

(b)

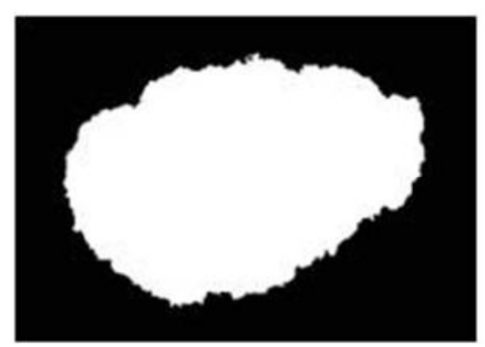

(c)

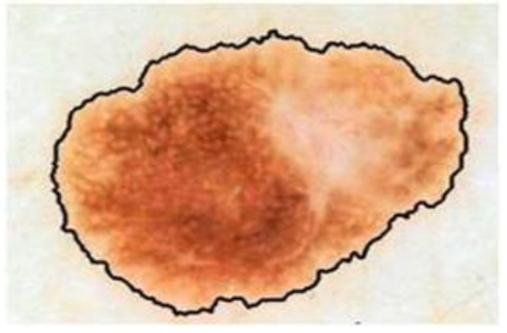

Fig. (2). Clark nevus with eccentric regression area. Results for the Boundary detection procedure: a) starting RGB image; b) binary mask; c) RGB image and superimposed contour.

computed, each pixel in the starting image can be directly labeled by taking into account the corresponding values for the two principal components. In particular assigning to each histogram region (or segment) an arbitrary intensity value, a gray-level image (or alternatively a false-color image) can be obtained where different regions are easily identified. An example is depicted in Fig. (3).

(ii) The last stage of the procedure for the automatic detection of blue whitish veil and regression is the classification of the regions constituting the lesion map as areas characterized by the presence of the criterion of interest. Consequently chromatic and morphological features have to be evaluated. In particular for each region the components of the corresponding pixels in the RGB, HSI (Hue, Saturation and Intensity) and CIE $L u v$ color spaces [16] are considered to compute mean value and standard deviation. A Logistic Model Tree (LMT) has been used for the classification [22] of the regions constituting the lesion map of the ELM image with respect to the blue veil and regression (LMT is a combination of a tree structure and logistic regression models resulting in a single tree according to the model proposed in [22]). For each one of the two criteria a different LMT is set-up.

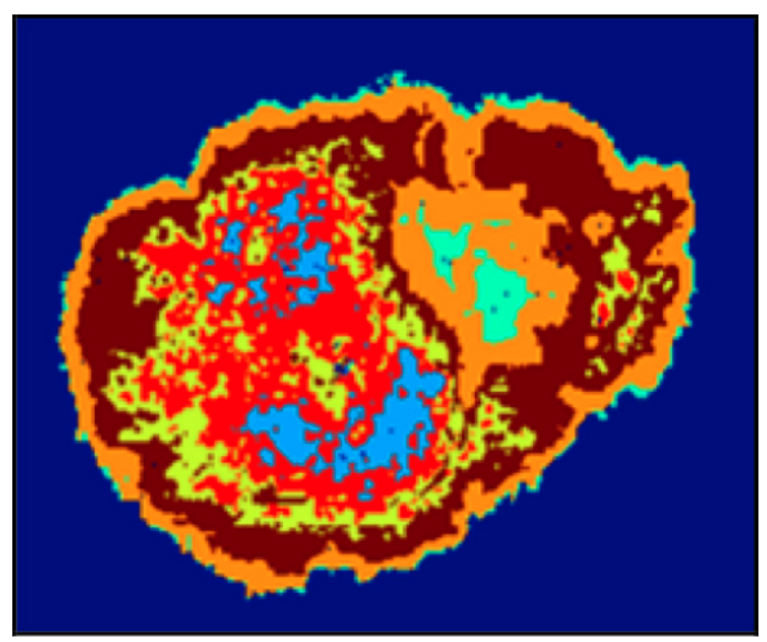

Fig. (3). Result of lesion segmentation. 


\section{Algorithm for the Detection of Irregular Streaks}

The presence of streaks or radial streaming defined as radially and asymmetrically arranged linear or bulbous extensions at the edge of the lesion, indicates histologically confluent radial junctional nests of melanocytes. Other works have looked at this uncommon subset of APN [4].

From an image processing point of view, these structures can be detected by searching for the simultaneous occurrence of two different structures: i) brown pigmentation localized in the same restricted region, and ii) finger-like track of the contour of the lesion. This process can be obtained analyzing the margins of the lesion split into 10 equally length segments. A color segmentation of the region of interest is performed in order to seek for the black/brown dermoscopic structures (see Fig. 4). Then a morphological irregularity index is computed and compared with a suitable threshold. The irregularity index is defined as "ratio of number of pixels constituting the lesion contour and the shortest path", where the "shortest path" pixels are the points belonging to the line that connects the farther contour points in the region.

\section{RESULTS AND DISCUSSION}

About the lesion segmentation and diagnosis, two subsets constituted by 110 and 100 ELM images have been adopted respectively as training and test sets. In particular,

- the Blue Veil is present in 70 and in 65 images of training and test set, respectively;

- $\quad$ the Regression is present in 40 and in 40 images of training and test set, respectively;

- Irregular streaks are present in 23 and in 30 images of training and test set, respectively.

In the following the experimental results are detailed for each parameter.

\section{Blue-Whitish Veil and Regression}

For each image of both the training and test set, the automatic lesion segmentation has been executed according to the proposed approach by considering 10 as maximum number of different color regions (segments). Then, each obtained lesion map has been analyzed in order to identify the main chromatic features corresponding to the single regions. Moreover, in order to achieve the decision rules to be applied to the test set, the lesion maps of the training set images have been inspected by the group of clinicians and a region based classification has been achieved with respect to the criteria of interest.

The Weka implementation [23] of the LMT algorithm has been adopted in order to obtain the predictive classifiers to be used in the automatic detection respectively of the Blue Veil and Regression.

About the lesion classification as whole with respect to the criterion of interest, the following strategy has been finally adopted: Blue Veil is detected within the lesion if the inner area obtained summing up the color segments classified as blue veil regions is wide enough (a threshold nearly equal to the $7 \%$ of the lesion area has been determined by a Receiver Operating Characteristic curve). Similar algorithm is also adopted for the detection of Regression (with a threshold equal to $5 \%$ of the lesion area). Following the previous strategies, very good results have been obtained in classifying the images of the test set. Fig. (5) shows some examples of corrected detection of blue-whitish veil (Fig. 5a-c) and regression areas (Fig. 5d-f).

The diagnostic performances on the test set are summarized in Table 1. More in detail, the Blue Veil has been correctly detected in 57 images against 5 lesions erroneously classified. Consequently scores equal to 0.87 and 0.85 have been achieved respectively for sensibility (ratio of corrected classification and total number of images characterized by the criterion of interest) and specificity (ratio of corrected classification and total number of images where the considered feature is absent). About the Regression structures, only 7 missed and 9 faulty detections have been obtained, thus resulting in sensibility and specificity both equal to 0.85 .

\section{Irregular Streaks}

The color segmentation of the regions is made with reference to only three classes, the darkest class is analyzed
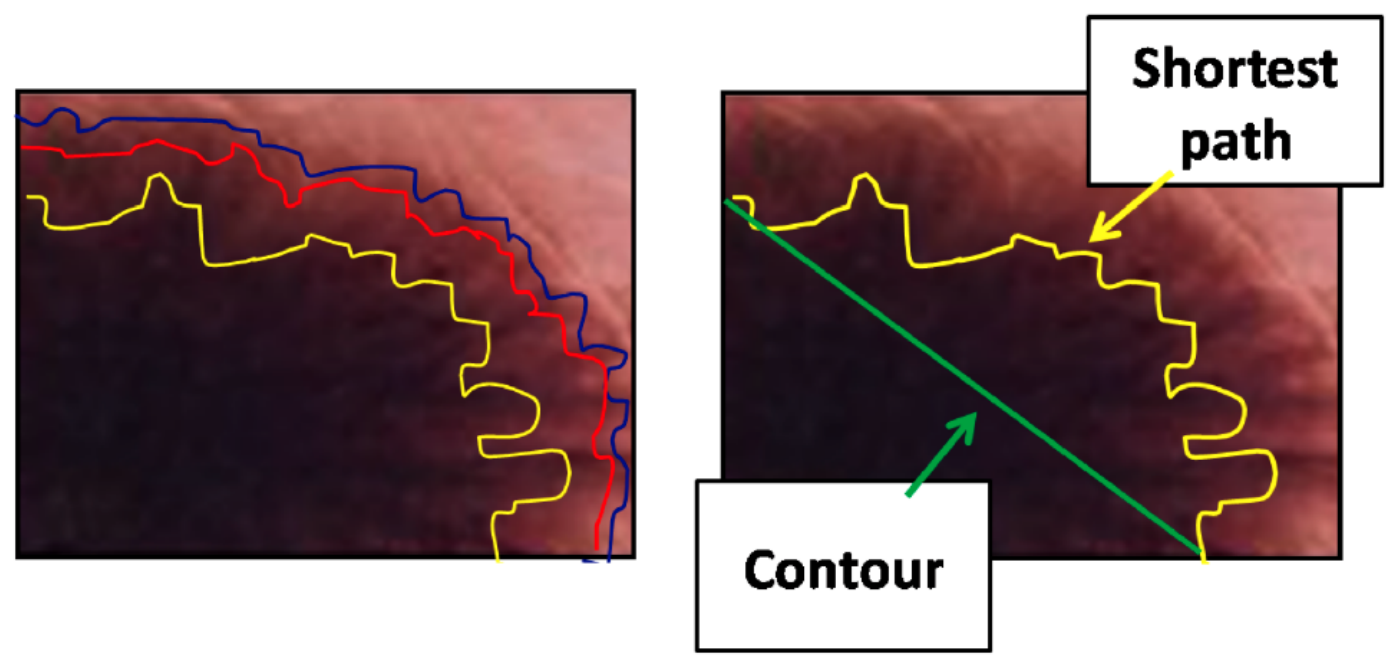

Fig. (4). Detection of irregular streaks. 
(a)



(d)

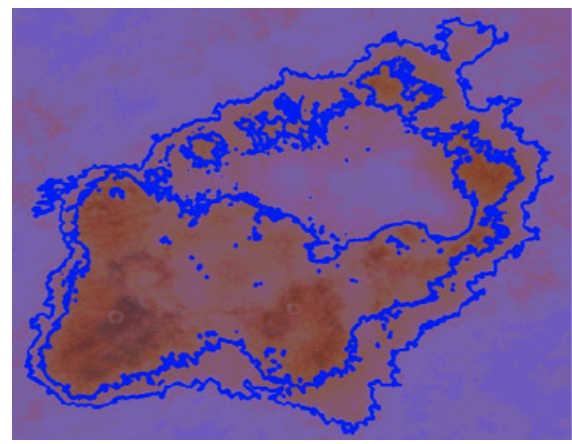

(b)

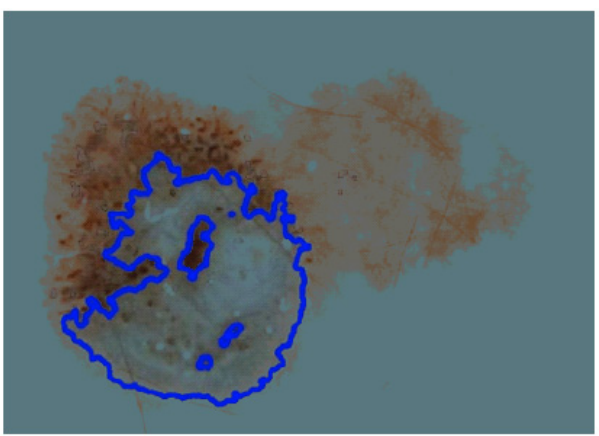

(e)

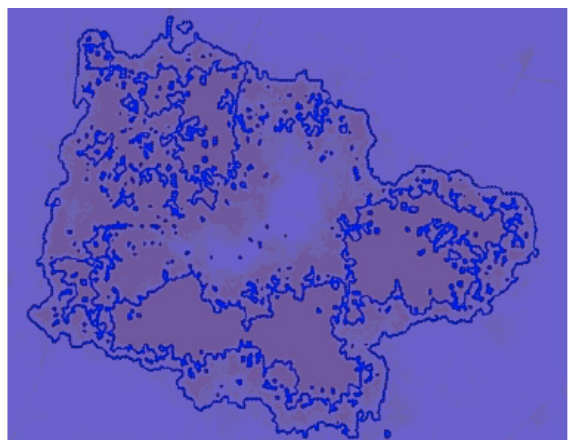

(c)



(f)

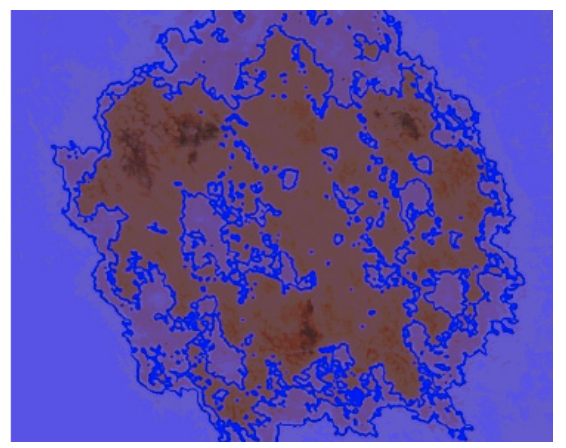

Fig. (5). Melanoma. Result of the automatic diagnosis: a-c) detection of blue whitish Veil; d-f) detection of regression area.



Fig. (6). Melanoma. Black/brown region identification.

with the aim to identify black/brown zone: the region is classified as dark if the average I value (of the HIS space) is less than a suitable threshold. For an example in Fig. (6), the classification results are reported, the blue contour zones are classified as dark the red ones not. Then the irregularity index is evaluated for each contour region and is compared with the threshold. Fig. (7) reports some examples of detected irregular streaks, signed in blue. Both thresholds, 0.23 and 1.4 respectively, have been determined by a Receiver Operating Characteristic curve (ROC curve) on the training image set (greater weight taken into account for sensibility). The obtained results on the test set are summarized in Table $\mathbf{1 .}$

Table 1. Experimental Results

\begin{tabular}{|c|c|c|}
\hline ELM Criterion & Sensibility (\%) & Specificity (\%) \\
\hline \hline Blue-whitish veil & 87 & 85 \\
\hline Irregular streaks & 86 & 88 \\
\hline Regression structures & 80 & 83 \\
\hline
\end{tabular}
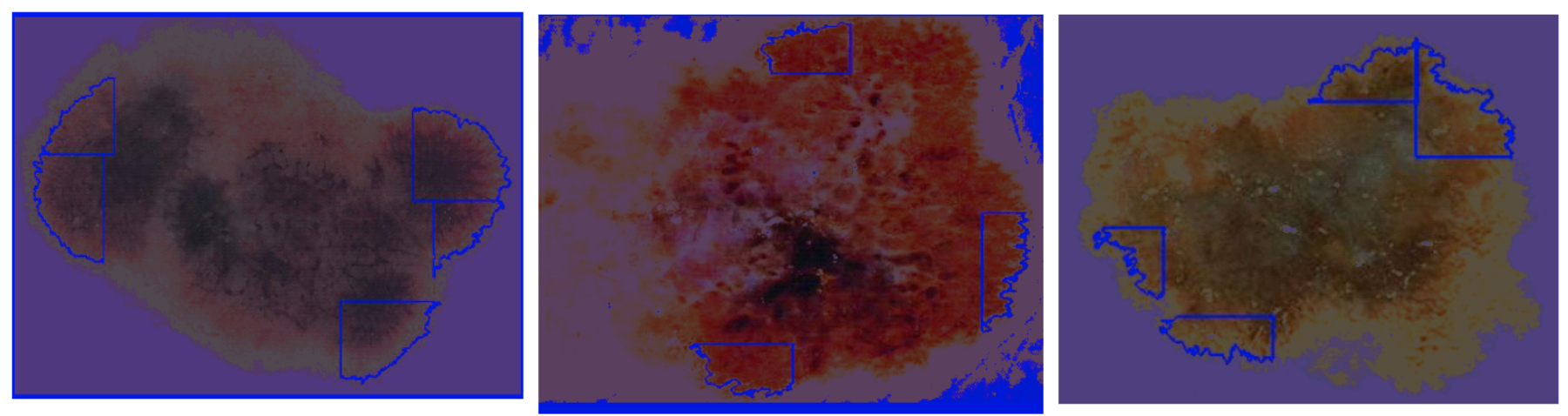

Fig. (7). Melanoma. Results of irregular streaks search. 


\section{REFERENCES}

[1] Leiter U, Buttner PG, Eigentler TK, Garbe C. Prognostic factors of thin cutaneous melanoma: an analysis of the central malignant melanoma registry of the German Dermatological Society. J Clin Oncol 2004; 22: 3660-7.

[2] Grimaldi L, Silvestri A, Brandi C, et al. Digital epiluminescence dermoscopy for pigmented cutaneous lesions, primary care physicians, and telediagnosis: a useful tool? J Plast Reconstr Aesthet Surg 2009; 62(8): 1054-8.

[3] Binder M, Schwarz M, Winkler A, et al. Epiluminescence microscopy: a useful tool for the diagnosis of pigmented skin lesion for formally trained dermatologists. Arch Dermatol 1995; 131(3): 286-91.

[4] Mayer J. Systematic review of the diagnostic accuracy of dermatoscopy in detecting malignant melanoma. Med J Aust 1997; 167: 206-10.

[5] Argenziano G, Soyer HP, Chimenti S, et al. Dermoscopy of pigmented skin lesions: results of a consensus meeting via the Internet. J Am Acad Dermatol 2003; 48(5): 679-93.

[6] Argenziano G, Fabbrocini G, Carli P, De Giorgi V, Sammarco E, Delfino M. Epiluminescence microscopy for the diagnosis of doubtful melanocytic skin lesions. Comparison of the ABCD rule of dermatoscopy and a new 7-point checklist based on pattern analysis. Arch Dermatol 1998; 134(12): 1563-70.

[7] Piccolo D, Ferrari A, Peris K, et al. Dermoscopy diagnosis by a trained clinicians $v s$ a clinician with minimal dermoscopy training vs computer-aided diagnosis of 341 pigmented skin lesions: a comparative study. Br J Dermatol 2002; 147: 481-6.

[8] Ganster H, Pinz A, Röhrer R, et al. Automated melanoma recognition. IEEE Trans Med Imaging 2001; 20(3): 233-9.

[9] Schmid P. Segmentation of digitized dermatoscopic images by two-dimensional color clustering. IEEE Trans Med Imaging 1999; 18(2): 164-71.

[10] Schmid-Saugeon P, Guillod J, Thiran JP. Towards a computeraided diagnosis system for pigmented skin lesions. Comput Med Imaging Graph 2003; 27(1): 65-78.

[11] Umbaugh SE, Moss RH, Stocker WV, Hance GA. Automatic color segmentation algorithms with application to skin tumor feature identification. IEEE Eng Med Biol Mag 1993; 12(3): 75-82.
[12] Grana C, Pellacani G, Cucchiara R, Seidenari S. A new algorithm for border description of polarized light surface microscopic images of pigmented skin lesions. IEEE Trans Med Imaging 2003; 22(8): 959-64.

[13] Burroni M, Corona R, Dell'Eva G, et al. Melanoma computer-aided diagnosis: reliability and feasibility study. Clin Cancer Res 2004; 10(6): 1881-6.

[14] Liguori C, Paolillo A, Pietrosanto A. An automatic measurement system for the evaluation of carotid intima-media thickness. IEEE Trans Instrum Meas 2001; 50(6): 1684-91.

[15] Levy A, Lindenbaum M. Sequential Karhuen-Loeve basis extraction and its Application to images. IEEE Trans Image Process 2000; 9(8): 1371-4.

[16] Ballard DH, Brown CM. Computer vision. New Jersey NJ: Prentice Hall 1982.

[17] Gonzalez RC, Woods RE. Digital image processing. New Jersey NJ: Prentice Hall 2002.

[18] Hoffmann K, Gambichler T, Rick A, et al. Diagnostic and neural analysis of skin cancer (DANAOS). A multicentre study for collection and computer-aided analysis of data from pigmented skin lesions using digital dermoscopy. Br J Dermatol 2003; 149(4): 801-9.

[19] Argenziano G, Soyer HP, De Giorgi V, et al. Interactive atlas of dermoscopy. Milan: EDRA Medical Publishing \& New Media 2002.

[20] Otsu N. A threshold selection method from gray-level histogram. IEEE Trans Syst Man Cybern 1979; 9(1): 62-6.

[21] Di Leo G, Fabbrocini G, Paolillo A, et al. Towards an Automatic Diagnosis System for Skin Lesions: Estimation of Blue-Whitish Veil and Regression Structures. In: Proceedings of the IEEE $6^{\text {th }}$ International Multi-Conference on System, Signals and Devices (SSD ’09), Djerba, Tunisia, March 2009; pp. 1-6.

[22] Landwehr N, Hall M, Frank E. Logistic Model Trees. In: Proceedings of the 14th European Conference on Machine Learning (ECML '03), Cavtat-Dubrovnik, Croatia, September 2003; pp. 241-252.

[23] Witten IH, Frank E. Data mining: practical machine learning tools and techniques. San Francisco: Morgan Kaufmann 2005. 